\title{
Antimicrobial Resistance in Outpatient Escherichia coli Urinary Isolates in Dakar, Senegal
}

\author{
Jean-Marie Sire, ${ }^{1}$ Pierre Nabeth, ${ }^{1}$ Jean-David Perrier-Gros-Claude, ${ }^{1}$ Ibrahim Bahsoun, ${ }^{1}$ \\ Tidiane Siby, ${ }^{2}$ Edgard Adam Macondo, ${ }^{2}$ Aïssatou Gaye-Diallo, ${ }^{3}$ Stéphanie Guyomard, ${ }^{4}$ \\ Abdoulaye Seck, ${ }^{1}$ Sébastien Breurec, ${ }^{1}$ Benoit Garin ${ }^{1}$ \\ ${ }^{1}$ Laboratoire de Biologie Médicale, Institut Pasteur, Dakar, Senegal; ${ }^{2}$ Laboratoire Bio24, Dakar, Senegal; ${ }^{3}$ Laboratoire de \\ Microbiologie, Hôpital Aristide Le Dantec, Dakar, Senegal; ${ }^{4}$ Laboratoire de Biologie, Hôpital Principal, Dakar, Senegal
}

\begin{abstract}
Background: Data regarding the evolution of antimicrobial resistance are needed to suggest appropriate empirical treatment of urinary tract infections (UTI) in developing countries. To assess the antimicrobial susceptibility of Escherichia coli, the predominant pathogen in community-acquired UTI, a prospective multicenter study was carried out in Dakar, Senegal.

Methodology: From February 2004 to October 2006, 1010 non-duplicate E. coli strains were collected from four centres. Antimicrobial susceptibility testing was performed using disk diffusion method according to the recommendations of the CA-SFM (2004).

Results: Most of the isolates were resistant to amoxicillin (73.1\%), amoxicillin-clavulanic acid (67.5\%), cephalothin (55.8\%), and trimethoprim/sulfamethoxazole (68.1\%). Extended spectrum beta-lactamase was detected in 38 strains. The overall resistance rates to nalidixic acid, norfloxacin and ciprofloxacin were $23.9 \%, 16.4 \%$ and $15.5 \%$, respectively. Most of the strains were susceptible to gentamicin, nitrofurantoin and fosfomycin (respective susceptibility rates, 93.8\%, 89.9\%, and $99.3 \%$ ). During this period, a significant decrease in sensitivity was observed for cephalothin, fluoroquinolones and trimethoprim/sulfamethoxazole $(p<0.001)$.

Conclusions: These data suggest that trimethoprim/sulfamethoxazole may no longer be used as empirical treatment for community-acquired UTI in Dakar. In order to preserve the activity of fluoroquinolones for future years, alternatives such as fosfomycin or nitrofurantoin should be considered.
\end{abstract}

Key Words: Escherichia coli, urinary tract infection, antimicrobial resistance, outpatient, Senegal.

J Infect Developing Countries 2007; 1(3):263-268.

Received 7 September 2007 - Accepted 14 November 2007.

Copyright (c) 2007 Sire et al. This is an open access article distributed under the Creative Commons Attribution License, which permits unrestricted use, distribution, and reproduction in any medium, provided the original work is properly cited.

\section{Introduction}

Symptomatic urinary tract infection (UTI) is one of the most commonly diagnosed infections in communities, particularly in otherwise healthy young women. Several studies conducted in Europe and in the USA have reported a steady increase of the resistance rate of uropathogens to commonly prescribed antibiotics (amoxicillin, trimethoprim-sulfamethoxazole), reducing therapeutic possibilities [1-3]. In some countries, resistance to fluoroquinolones is also emerging [4]. In developing countries, the frequently irrational use of antibiotics predicts a worrisome increase in the prevalence of antimicrobial resistance.

Worldwide, Escherichia coli has been established as the most commonly isolated pathogen among organisms implicated in community-acquired UTI. In Senegal the same situation prevails [5]. Hence, a prospective threeyear study was undertaken, from February 2004 to October 2006, to determine the susceptibility patterns of $E$. coli strains isolated from outpatients referred from four clinical laboratories located in Dakar, the capital of Senegal.

\section{Materials and Methods \\ Patients}

From February 2004 to October 2006, a total of 1010 E. coli strains isolated from the urine of patients with community-acquired urinary tract infections was collected from four clinical laboratories in Dakar. Two of these laboratories (Institut Pasteur, Bio24) are private institutions receiving patients referred by general practitioners. The two others are located inside hospitals, one being a major teaching hospital (Hôpital Aristide Le 
Dantec) and the other a military hospital (Hôpital Principal). These hospital laboratories recruited subjects examined in the outpatients department with a suspected diagnosis of UTI. The patients attending the private laboratories, as well as the military hospital, belonged to upper and middle classes, while the majority of samples examined in the teaching hospital belonged to patients from the underprivileged classes. Pregnant women aged between 15 and 45 years were recorded. Patients were questioned about some specific conditions (i.e. hospitalization, antibiotic treatment, UTI and urinary catheterization) that could have occurred during the month preceding the onset of the symptoms. The nature of previously prescribed drugs was not included in the statistical analysis because very few patients were able to name the prior antibiotic used precisely.

\section{Bacterial strains}

Leukocyte count, isolation and identification of bacteria were conducted on site from urine samples by all the laboratories as part of their routine diagnostic. Only a pure culture of $E$. coli with a colony count of $\geq 10^{5} \mathrm{cfu} / \mathrm{ml}$ associated with pyuria (WBC $\geq 10^{4} / \mathrm{ml}$ ) was considered significant for inclusion. All $E$. coli isolates were collected for antimicrobial susceptibility testing by the coordinating laboratory located in Institut Pasteur. Species identification was checked by inoculation onto a culture medium allowing differentiation of main uropathogens (Chromagar orientation medium, Becton-Dickinson, Heidelberg, Germany). According to the manufacturer's instructions, indole-positive, pink-coloured colonies were confirmed as $E$. coli. In case of uncertainty, clear identification was performed using API 20E gallery (bioMérieux, Marcy l'Etoile, France).

\section{Antimicrobial susceptibility testing}

Antibiotics' susceptibility was performed by disk diffusion method according to the guidelines of the Antibiogram Committee of the French Society for Microbiology (CA-SFM) [6]. Antibiotic disks were obtained from Bio-Rad laboratories (Marnes-la-Coquette, France) and strains were tested against the following antimicrobial agents (quantity of antibiotic per disk): amoxicillin $(10 \mu \mathrm{g})$, amoxicillin-clavulanic acid $(20 \mu \mathrm{g} / 10 \mu \mathrm{g})$, ticarcillin $(75 \mu \mathrm{g})$, cephalothin $(30 \mu \mathrm{g})$, cefotaxime $(30 \mu \mathrm{g})$, ceftazidime $(30 \mu \mathrm{g})$, nalidixic acid $(30 \mu \mathrm{g})$, norfloxacin (5 $\mu \mathrm{g})$, ciprofloxacin (5 $\mu \mathrm{g})$, trimethoprim/sulfamethoxazole (cotrimoxazole) (1.25 $\mu \mathrm{g} / 23.75 \mu \mathrm{g})$, gentamicin $(15 \mu \mathrm{g})$, nitrofurantoin $(300 \mu \mathrm{g})$, and fosfomycin $(50 \mu \mathrm{g})$. Extended spectrum beta-lactamase (ESBL) producing organisms were detected by the doublediffusion method looking for synergy images between amoxicillin-clavulanic acid and cefotaxime, or ceftazidime disks. Isolates producing ESBL were considered to be resistant to cefotaxime and ceftazidime according to the CASFM recommendations. Inhibition zone diameters were measured with an automated zone size reader (Osiris, Bio-Rad) and isolates were classified as susceptible, intermediate or resistant according to the annual report of the CA-SFM [6]. Intermediate and resistant strains were further grouped together in the resistant group for analysis. E coli ATCC 25922 was used as the control strain.

\section{Statistical analysis}

Statistical analysis was performed using STATA software version 6.0. Risk factors predicting $E$. coli resistance were estimated by univariate analysis and tested when appropriate by $\chi^{2}$ or Fisher's exact test. Variables with $\mathrm{P}<0.25$ in the univariate analysis were then included in a multivariable logistic regression model. Variables with an adjusted odds ratio of $\mathrm{P}<0.05$ were considered as risk factors.

\section{Results}

One thousand and ten non-duplicate $E$. coli strains (561 from Institut Pasteur, 225 from Bio24, 177 from the teaching hospital laboratory, 47 from the military hospital laboratory), were collected during the study period. Seven hundred and seventy strains $(76.3 \%)$ were isolated from women and 239 (23.7\%) from men (sex status unknown for 1 patient). Patients were classified in three categories according to age: patients under 15 years $(115 ; 11.5 \%)$, patients aged from 15 to 50 years $(635 ; 63.6 \%)$ and patients over 50 years $(249 ; 24.9 \%)$, data was not available for 11 patients $(1.1 \%)$. Among women aged from 15 to 45 years, $16.7 \%(84 / 504)$ were pregnant. The percentage of patients presenting potential risk factors for antimicrobial resistance was recorded as follows: hospitalization $(4.6 \%$, data reported for 940 patients), antibiotic exposure $(16.4 \%$ of 971 
patients), UTI (9.6\% of 948 patients) and urinary catheterization (2.4\% of 918 patients). Missing data were due to the difficulty in collecting oral information from some patients.

Resistance rates of $E$. coli strains to selected antimicrobial agents by year of study are presented in Table 1.

Table 1. Antimicrobial resistance of $E$. coli isolates from community acquired urinary tract infections by antibiotic and year of study.

\begin{tabular}{lllll} 
& \multicolumn{4}{l}{ No. of isolates resistant to antibiotics $(\%)$} \\
\cline { 2 - 5 } Year (no. & 2004 & 2005 & 2006 & Total \\
isolated) & $(289)$ & $(449)$ & $(272)$ & $(1010)$ \\
\hline \multirow{2}{*}{ AMX } & 208 & 323 & 207 & 738 \\
& $(72.0)$ & $(71.9)$ & $(76.1)$ & $(73.1)$ \\
AMC & 186 & 302 & 194 & 682 \\
& $(64.4)$ & $(67.3)$ & $(71.3)$ & $(67.5)$ \\
TIC & 190 & 307 & 190 & 687 \\
& $(65.7)$ & $(68.4)$ & $(69.9)$ & $(68.0)$ \\
CF & 134 & 254 & 176 & 564 \\
& $(46.4)$ & $(56.6)$ & $(64.7)$ & $(55.8)$ \\
CTX & 8 & 13 & 14 & 35 \\
& $(2.8)$ & $(2.9)$ & $(5.2)$ & $(3.5)$ \\
CAZ & 10 & 15 & 13 & 38 \\
& $(3.5)$ & $(3.3)$ & $(4.8)$ & $(3.8)$ \\
NA & 58 & 110 & 73 & 241 \\
& $(20.1)$ & $(24.5)$ & $(26.8)$ & $(23.9)$ \\
NOR & 31 & 72 & 63 & 166 \\
& $(10.7)$ & $(16.0)$ & $(23.2)$ & $(16.4)$ \\
CIP & 28 & 69 & 60 & 157 \\
& $(9.7)$ & $(15.4)$ & $(22.1)$ & $(15.5)$ \\
SXT & 169 & 323 & 196 & 688 \\
& $(58.5)$ & $(71.9)$ & $(72.1)$ & $(68.1)$ \\
GE & 16 & 22 & 25 & 63 \\
& $(5.5)$ & $(4.9)$ & $(9.2)$ & $(6.2)$ \\
FUR & 27 & 45 & 30 & 102 \\
& $(9.5)$ & $(10.0)$ & $(11.0)$ & $(10.1)$ \\
FOS & 4 & 1 & 2 & 7 \\
& $(1.4)$ & $(0.2)$ & $(0.7)$ & $(0.7)$ \\
\hline
\end{tabular}

AMX, amoxicillin; AMC, amoxicillin-clavulanic acid; TIC, ticarcillin; CF, cephalothin; CTX cefotaxime; CAZ, ceftazidime; NA, nalidixic acid; NOR, norfloxacin; CIP ciprofloxacin; SXT, trimethoprim/sulfamethoxazole (cotrimoxazole); GE, gentamicin; FUR, nitrofurantoin; FOS fosfomycin.

The majority of the isolates were resistant to amoxicillin, amoxicillin-clavulanic acid, ticarcillin and first generation cephalosporins (respective overall resistance rates: $73.1 \%, 67.5 \%, 68 \%$ and $55.8 \%$ ). Third generation cephalosporins (cefotaxime, ceftazidime) remained active in most cases (respective overall resistance rates: $3.5 \%$ and $3.8 \%$ ). ESBL was detected in 38 strains (3.8\%): 14 strains in 2004 (4.8\%), 12 strains in 2005 (2.7\%), and 12 strains in 2006 (4.4\%). A high resistance rate was found for cotrimoxazole (68.1\%). As for quinolones, $23.9 \%$ of the strains were resistant to nalidixic acid, $15.5 \%$ to ciprofloxacin and $16.4 \%$ to norfloxacin. Most of the strains were susceptible to gentamicin $(93.8 \%$ susceptible) and nitrofurantoin (89.9\% susceptible). Almost all strains were susceptible to fosfomycin (99.3\% susceptible).

During this study, a significant increase was observed for resistance to cephalothin $(P<0.001)$, norfloxacin $(P<0.001)$, ciprofloxacin $(P<0.001)$ and cotrimoxazole $(P<0.001)$. According to multivariate analysis (Table 2), age $>50$ years was identified as a risk factor for resistance to nalidixic acid, norfloxacin and ciprofloxacin.

Table 2. Variables statistically related to antimicrobial resistance in the multivariate model.

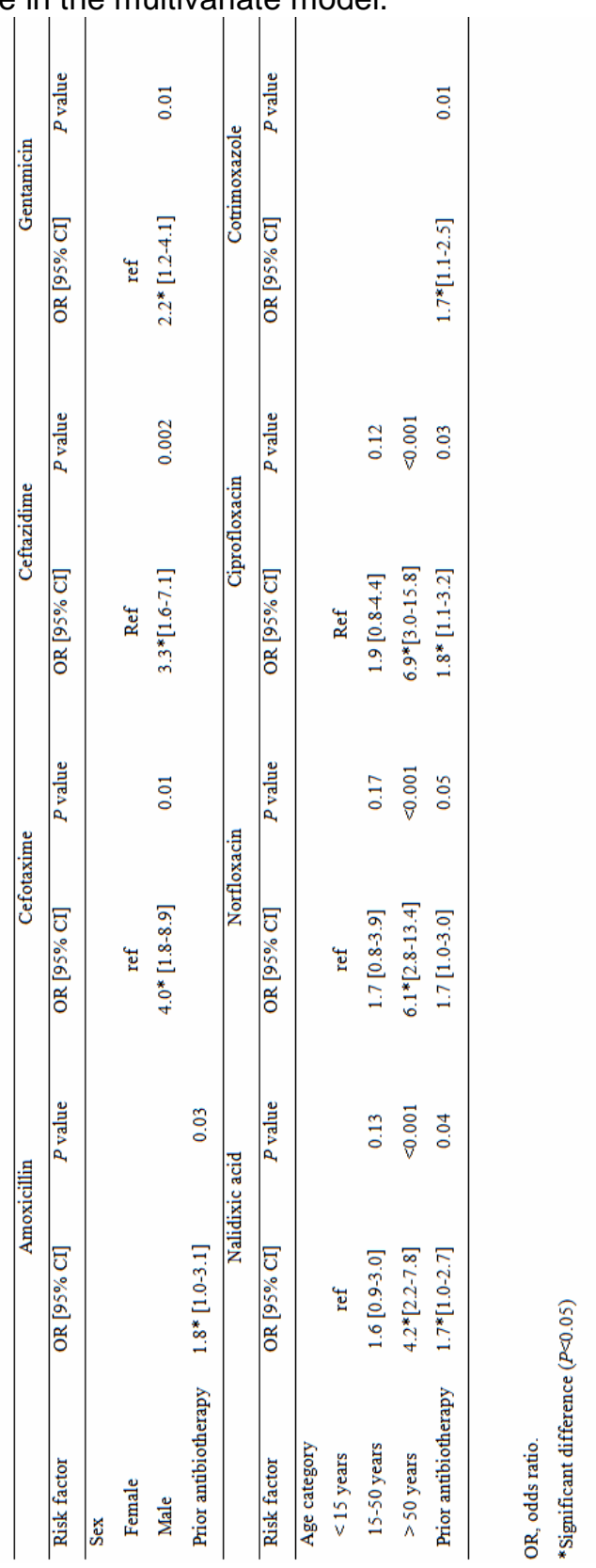


Male gender was significantly linked to a higher rate of resistance to gentamicin and third generation cephalosporins (cefotaxime, ceftazidime). Antibiotic exposure within the month before infection was significantly associated with resistance to amoxicillin, nalidixic acid, ciprofloxacin, cotrimoxazole and approached significant resistance to norfloxacin $(P=0.05)$. Within the same period, previous hospitalization, prior UTI, and prior urinary catheterization were not considered in our model to be risk factors of antimicrobial resistance to any of the tested drugs.

\section{Discussion}

Antimicrobial susceptibility testing of uropathogens is known to overestimate rates of resistance because antibiograms are performed mostly if empirical treatment fails, or if patients have underlying factors [7]. In developing countries, this bias may be more present because many patients cannot afford medical expenses, so they undergo urine analysis only in case of repeated or complicated UTI. Nevertheless, this study confirms that levels of antimicrobial resistance of outpatient $E$. coli urinary isolates to aminopenicillins, cotrimoxazole and fluoroquinolones are higher in Dakar than those observed in Europe and North America [1,2]. This is consistent with recent data reported in other developing countries [8-11].

Previous data collected in our laboratory $[5,12,13]$ confirm the steady decrease in susceptibility of $E$. coli strains isolated from community-acquired UTI to amoxicillin-clavulanic acid, cephalothin, quinolones, and cotrimoxazole since 1999. To date, cotrimoxazole is still recommended by Senegalese health authorities as the first line treatment for UTI in primary health care structures, which can explain the high level of resistance rate observed in this study. The significant increase of resistance to quinolones, and the high resistance rates reached in 2006, are worrisome and could be related to the easy access and the misuse of this class of antibiotics in Senegal [13]. Another factor could be the generalized use of fluoroquinolones in animal feed in the country (especially in poultry intensive breeding), and the subsequent transmission of resistant strains from animals to humans [14].

Accuracy of the risk factors analysis must be put into perspective because information was obtained directly from patients without checking their medical reports. Nevertheless, multivariate analysis highlighted some specific risk factors. Antibiotic exposure a month before infection was reported by multivariate analysis as a risk factor for resistance to amoxicillin, nalidixic acid, ciprofloxacin, and cotrimoxazole. This data partially confirms a previous study conducted in our laboratory, when antibiotic exposure within the previous six months was found associated with resistance to amoxicillin, amoxicillin-clavulanic acid and cotrimoxazole [13]. In our study, age over 50 years and male gender were found statistically significant with resistance to fluoroquinolones by univariate analysis $(P<0.01)$. This result has been previously reported and could be related to the tendency for male or elderly patients to be infected by more resistant strains because they are more likely to present complicated urinary tract infections [15]. In our multivariate model, if age > 50 years remained independently associated with resistance to fluoroquinolones $(\mathrm{P}<0.001)$, male gender did not. In addition, a significant relationship has been found between male gender and resistance to third generation cephalosporins and gentamicin. To our knowledge, association to male gender has not been previously recorded for these antibiotics.

Aminopenicillins or cotrimoxazole should no longer be used as empirical treatments of UTI in Senegal. Alternatives must be recommended, especially for empirical treatments of uncomplicated UTI (cystitis) in women. Although most $E$. coli isolates were susceptible to nitrofurantoin, this antimicrobial agent has demonstrated poor in vitro activity against Enterobacteriaceae other than E. coli [16]. Moreover, this antibiotic does not penetrate into tissues and could not be used to treat infections with suspected tissue involvement (such as pyelonephritis). Generalized prescription of fluoroquinolones as empirical treatment for cystitis should be abandoned. This common practice facilitates the emergence of strains resistant to this class of antibiotics and promotes the emergence of multidrug-resistant strains in the community [17]. A substantial effort has to be made in Senegal to protect the activity of fluoroquinolones by controlling their prescription in human medicine and limiting (or even banning) their use in animal husbandry. 
A previous study conducted in Dakar has shown that resistance rates of Enterobacteriaceae to fosfomycin-trometamol, a form of fosfomycin characterized by a high oral biodisponibility, was very low [18]. Our data about E. coli confirm these findings. Fosfomycin-trometamol has been proposed as a reliable empirical treatment for uncomplicated UTI because of its easy use (single dose), its good tolerance, and its efficacy [19]. Although in vitro resistance to fosfomycin is easily acquired by multiple one-step mutations, treatment failures are rare. A recent study suggests that the biological fitness of these resistant mutants is affected, so they are less likely to infect the bladder [20]. Unfortunately, this antibiotic is not yet available in Senegal. However, to preserve the efficacy of fluoroquinolones, we suggest that fosfomycin could be an option for empirical treatment of urinary tract infections in outpatients, and should be introduced into the national guidelines.

\section{Acknowledgements}

Special thanks to Rokhaya Mbaye for her technical assistance. This study was supported by the French Ministry of Foreign Affairs as part of a special grant dedicated to the creation of an international network for surveillance and analysis of antimicrobial resistance.

\section{References}

1. Kahlmeter G (2003) An international survey of the antimicrobial susceptibility of pathogens from uncomplicated urinary tract infections: the ECO-SENS project. J Antimirobial Chemother 51: 69-76.

2. Zhanel GG, Hisanaga TL, Laing NM et al (2006) Antibiotic resistance in Escherichia coli outpatients urinary isolates: final results from the North American Urinary Tract Infection Collaborative Alliance (NAUTICA). Int J Antimicrob Agents 27:468-475.

3. Gupta K, Scholes D, Stamm WE (1999) Increasing prevalence of antimicrobial resistance among uropathogens causing acute uncomplicated cystitis in women. JAMA 281:736-738.

4. Alós JI, Serrano MG, Gómez-Garcés JL, Perianes J (2005) Antibiotic resistance of Escherichia coli from community-acquired urinary tract infections in relation to demographic and clinical data. Clin Microbiol Infect 11:199-203

5. Dromigny JA, Nabeth P, Perrier-Gros-Claude JD (2002) Distribution and susceptibility of bacterial urinary tract infections in Dakar, Senegal. Int J Antimicrob Agents 20:339-347.

6. CA-SFM Comité de l'Antibiogramme de la Société Française de Microbiologie: Communiqué 2006 (Edition de janvier 2006). Available from URL: http://www.sfm.asso.fr.

7. Heginbothom ML et al. (2004) Laboratory testing policies and their effects on routine surveillance of community antimicrobial resistance. $\mathrm{J}$ Antimicrobial Chemother 53:1010-1017.

8. Hima-Lerible H, Ménard D, Talarmin A (2003) Antimicrobial resistance among uropathogens that cause community-acquired urinary tract infections in Bangui, Central African Republic. J Antimicrobial Chemother 51:192-194.

9. Atif $A A$, Osman $H$, Mansour AM, Musa $H A$, Ahmed AB, Karrar Z, Hassan HS (2000) Antimicrobial agent resistance in bacterial isolates from patients with diarrhea and urinary tract infection in the Sudan. Am J Trop Med Hyg 63:259-263.

10. Debasis B, Pratima G, Ramjee P, Vikram S, Muktanjali A, Ashish K (2006) Choice of antibiotic for empirical therapy of acute cystitis in a setting of high antimicrobial resistance. Indian J Med Sci 60:53-58.

11. Matute AJ et al. (2004) Resistance of uropathogens in symptomatic urinary tract infections in León, Nicaragua. Int J Antimicrob Agents 2004 23:506-509.

12. Dromigny JA, Ndoye $B$, Macondo EA, Nabeth $P$, Siby $T$, Perrier-Gros-Claude JD (2003) Increasing prevalence of antimicrobial resistance among Enterobacteriacae uropathogens in Dakar, Senegal: a multicenter study. Diagn Microbiol Infect Dis 47:595-600.

13. Dromigny JA, Nabeth $P$, Juergens-Behr A, Perrier-GrosClaude JD (2005) Risk factors for antibiotic-resistant Escherichia coli isolated from community-acquired urinary tract infections in Dakar, Senegal. J Antimicrobial Chemother 56:236-239.

14. Miller LG, Tang AW (2004) Treatment of uncomplicated urinary tract infections in an era of increasing antimicrobial resistance. Mayo Clin Proc 79:1048-1054.

15. Sahm DF, Thornsberry C, Mayfield DC, Jones ME, Karlowsky JA. (2001) Multidrug-resistant urinary tract isolates of Escherichia coli: prevalence and patient demographics in the United States in 2000. Antimicrob Agents Chemother 45:1402-1406.

16. Farrell DJ, Morrissey I, De Rubeis D, Robbins $M$, Felmingham D (2003) A UK multicentre study of the antimicrobial susceptibility of bacterial pathogens causing urinary tract infections. J Infect 46:94-100.

17. Karlowsky JA, Hoban DJ, DeCorby MR, Laing NM, Zhanel GG (2006) Fluoroquinolone-resistant urinary isolates of Escherichia coli from outpatients are frequently multidrug resistant: results from the North American Urinary Tract Infection Collaborative Alliance-Quinolone Resistance study. Antimicrob Agents Chemother 50:2251-2254.

18. Nabeth $P$, Perrier-Gros-Claude JD, Juergens-Behr A, Dromigny JA (2005) In vitro susceptibility of quinoloneresistant Enterobacteriacae uropathogens to fosfomycintrometamol, in Dakar, Senegal. Scand J Inf Dis 37:497499.

19. Lobel B (2003) Short term therapy for uncomplicated urinary tract infections today. Clinical outcome upholds the theories. Int J Antimicrob Agents 22(Suppl 2):85-87.

20. Nillson AI, Berg OG, Aspevall O, Kahlmeter G, Andersson DI (2003) Biological costs and mechanisms of fosfomycin resistance in Escherichia coli. Antimicrob Agents Chemother 47:2850-2858. 
Corresponding Author: Jean-Marie Sire, Tel: +22133 83992 31; Fax: +221 3382270 52; E-mail address: sire@pasteur.sn.

Conflict of Interests: The authors declare that they have no conflict of interests. 